\title{
A Two-Dimensional Fault Diagnosis Model of Induction Motors using a Gabor Filter on Segmented Images
}

\author{
Jia Uddin ${ }^{1}$, Md. Rashedul Islam ${ }^{2}$, Jong-Myon Kim ${ }^{3, *}$, Cheol-Hong Kim ${ }^{4}$ \\ ${ }^{1}$ Department of Computer Science and Engineering, BRAC University, 45 \\ Mohakhali, \\ Dhaka 1212, Bangladesh \\ ${ }^{2,3}$ Department of Electronic/Electrical and Computer Engineering, School of \\ Electrical Engineering, University of Ulsan, De 93 Daehak-ro, Nam-gu, Ulsan \\ 680749, Korea \\ ${ }^{4}$ School of Electronics and Computer Engineering, Chonnam National University, \\ Gwangju, Korea \\ jia.uddin@bracu.ac.bd, \{rashed.cse,jongmyon.kim,cheolhong\}@gmail.com
}

\begin{abstract}
Image segmentation has received extensive attention due to the use of high-level descriptions of image content. This paper proposes a fault diagnosis model using a Gabor filter on segmented two-dimensional (2D) gray-level images. The proposed approach first converts time domain $A E$ signals into $2 D$ gray-level images to exploit texture information from the converted images. $2 D$ discrete wavelet transform $(D W T)$ is then applied to select appropriate (vertical) texture information and reconstructed it into an image. The reconstructed image is segmented into a number of sub-images depending on the segment size and a Gabor filter is applied on each sub-image. Finally, feature vectors are extracted from the Gabor-filtered sub-images and utilized as inputs in a one-against-all multiclass support vector (OAA-MCSVM) to identify each fault in an induction motor. In this study, multiple bearing defects under various segment sizes are utilized to validate the effectiveness of the proposed method. Experimental results indicate that the proposed model outperforms conventional Gabor-filter-based 2D fault diagnosis algorithms in classification accuracy, exhibiting a $97 \%$ average classification accuracy for $64 \times 64$ segmented images.
\end{abstract}

Keywords: Fault diagnosis, induction motor, acoustic emission, Gabor filter

\section{Introduction}

With advances in manufacturing has come increased demand for higher production rates, improved product quality, and larger throughput volumes. The induction motor is one of the most significant elements in a variety of industrial applications. An unexpected failure of the induction motor may cause significant economic losses by interrupting the production rate. An effective state monitoring system is critical for reducing degradation in machine performance by detecting and acting in a reliable and timely manner [1]. Therefore, fault detection and diagnosis of induction motors is the subject of intensive research.

Current and voltage signals have been utilized in research for easy signal measurement and low-cost monitoring systems [2-5]; however, these signals contain unnecessary components (characteristic harmonics) that typically cause signal distortion. Vibration analysis has been frequently used to diagnosis induction motor defects because it provides

${ }^{*}$ Corresponding author. 
the most intrinsic information about multiple faults using higher energy levels [6-11]. However, when compared with vibration signals, acoustic emission (AE) signals can improve diagnosis accuracy by inhibiting noise interference with a high-frequency spectrum [12]. It also has better capacity for early detection of fault propagation than with the vibration signal. Thus, the use of AE signals in state monitoring of an induction motor is a preferred alternative.

A number of diagnosis methods have been developed and are currently in use, including power spectrum estimation, fast Fourier transform, and envelope spectrum analysis. These methods are based on the assumption of stationary and linearity of input signals, so they are not viable for a dominant role in non-linear and non-stationary signals analysis. Several techniques have been proposed to analyze non-stationary and non-linear fault signals, including short-time Fourier transform (STFT), wavelet transform (WT), Wigner-Ville distribution (WVD), Hilbert Huang transform (HHT), and Teiger-Huang transform (THT). The limitation between time and frequency resolutions is one of the major constraints in STFT. Although the WT has exhibited improved performance over the STFT, it is non-adaptive in nature due to its uniform resolution for all scales. The WVD of discrete time signals suffers from aliasing problems, while HHT/THT is adaptive with input signals and can select intrinsic mode function (IMF) based on the input signals; however, selecting the number of IMFs based on the threshold value is a limiting factor in HHT and THT [9].

In recent years, two-dimensional (2D) representations of signals have been utilized in various studies of fault diagnosis [7, 13-17], where time-domain signals are converted to 2D gray-level images to exploit the texture information from the converted images. In [13], Do et al. proposed a fault diagnosis model using a 2D feature of mechanical signals utilizing scale invariant feature transform (SIFT). The drawback of this method is that the accuracy of extracting frequency information is limited by the length of the window relative to the duration of the analyzed signal. In [15], Jang et al. proposed a fault classification method using texture analysis, where the gray-level co-occurrence matrix (GLCM) was used as a feature extraction method. However, during construction of the GLCM matrix from a 2D gray level image, the texture information may distort, significantly impacting the properties of feature vectors. In [7], Dinh et al. proposed a 2D fault diagnosis model using the significant pixels of an image in texture analysis. The selection of a threshold value and the order of Gaussian radial functions significantly control the number of dominant pixels. Kang et al. proposed a new 2D representation technique to observe the clean texture for each fault [18]; this method uses wavelet coefficients deduced from the Shannon mother wavelet function with varying dilation and translation parameters to generate 2D gray-level images, and a global neighborhood structure (GNS) map to extract the texture features of each fault. Since time and frequency components were considered in the $2 \mathrm{D}$ translation process, we expected to extract the distinguishable texture for each fault; however, the robustness of the new translation method was not justified with the necessary experimental results. In [14], Uddin et al. proposed a 2D fault diagnosis model using GNS/LNS (Local Neighborhood Structure) maps to extract the texture feature vectors. The major drawback of this model is the non-adaptive selection of window sizes in the construction of feature vectors. In [16], Rifat et al. proposed a 2D texture feature-extraction-based fault diagnosis model utilizing a local binary pattern algorithm to extract the texture feature vectors; however, due to the non-adaptive selection of the number of neighbor pixels and radius of the LBP operator, this method is not robust for the extraction of features from the images. An efficient image representation approach was proposed in $[19,20]$ using a Gabor filter. Gabor filters possess a number of interesting mathematical properties including a smooth and indefinitely differentiable shape, a lack of side lobes in both the space and frequency domains, and the generation of high-dimensional representations. 
When compared to conversional 1D fault diagnosis models, the texture image conversion in a $2 \mathrm{D}$ fault diagnosis has the additional advantages of reduced noise effects and the use of small input signals; however, the extraction of distinct textures for each non-stationary and non-deterministic fault signal is still under research [21]. These drawbacks have motivated research into a new 2D fault diagnosis model using a Gabor filter on segmented images.

In this paper, a $2 \mathrm{D}$ texture image of a time-domain $\mathrm{AE}$ signal is segmented into a number of equally-sized sub-images. A 2D Gabor filter with various scales and frequencies is applied on each sub-image, where the size of the Gabor-filtered image is equal to the size of the sub-images. Finally, feature vectors are extracted from the Gaborfiltered sub-images and utilized as inputs for a multiclass support vector machine to identify each fault of an induction motor.

The rest of the paper is organized as follows: Section 2 describes the proposed diagnosis methodology, and Section 3 presents a test fixture for the experiments, introduces multiple bearing defects for diagnosis in this study, and validates the effectiveness of the proposed model in terms of classification accuracy. Finally, Section 4 concludes this paper.

\section{Proposed Fault Diagnosis Methodology}

The proposed model consists of the following five blocks: (a) the conversion of 1D time-domain AE signals to 2D gray images, (b) the application of 2D-DWT for selecting appropriate (vertical) texture information, and then the reconstruction of this information in a 2D image, (c) the generation of a number of sub-images of the reconstructed image depending on segment size, and the application of a 2D Gabor filter on each sub-image, (d) the extraction of statistical features from the sub-images, and finally, (d) the training and testing of the SVMs with feature vectors to classify multiple faults. Figure 1 illustrates a block diagram of the proposed fault-diagnosis model using a $2 \mathrm{D}$ Gabor filter on segmented images.

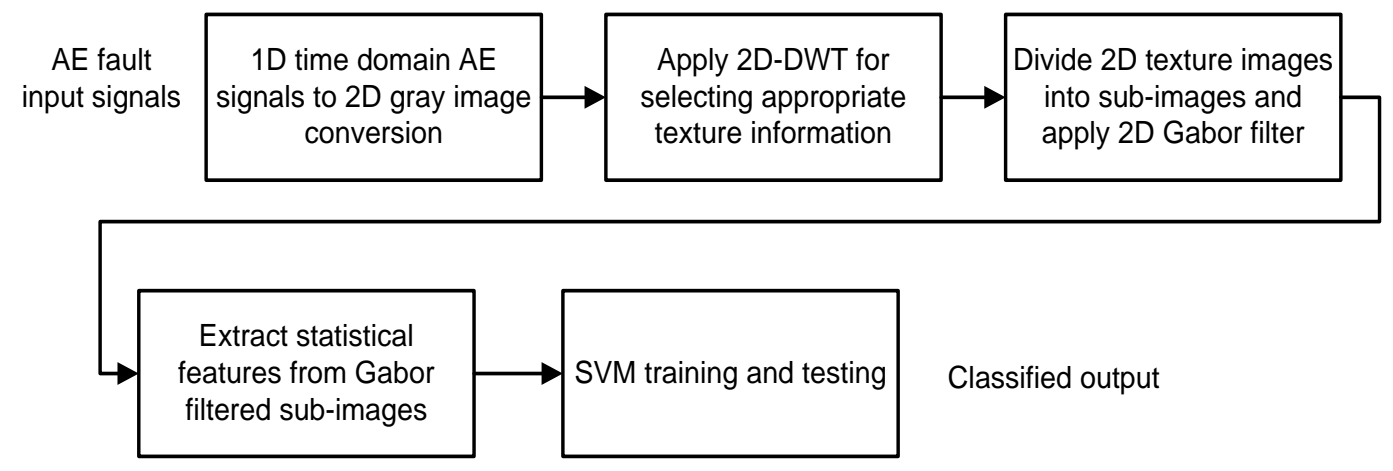

\section{Figure 1. Block Diagram of the Proposed Fault-diagnosis Model using a 2D Gabor Filter on Segmented Images}

In this study we utilize a 2D space conversion to improve feature reliability and reduce noise effects. The samples of a time-domain signal are utilized to generate a gray-level image. A detailed conversion process of a time-domain signal to a $2 \mathrm{D}$ gray-level image is presented in Figure 2, where a time-domain AE fault signal is divided into a number of subparts. The amplitude of a sample of each subpart becomes the intensity of a pixel in 2D space, and all the sample values of each sub-band are placed in a column of 2D space. Finally, a 2D gray-level image is generated by normalizing the converted 2D space with a size of $\mathrm{N} \times \mathrm{M}$, where $\mathrm{N}$ is the number of samples in a sub-band and $\mathrm{M}$ is the number of sub-bands. In our experiment, we set the sample size of each fault signal at 65536 and 
divide each time-domain signal into 256 sub-parts, where each part has 256 samples. By arranging the samples of each sub-part in a column, we generate a 2D matrix with a size of $256 \times 256$. At the end, a gray-level texture image is generated by normalizing on a scale of 0 to 255 .

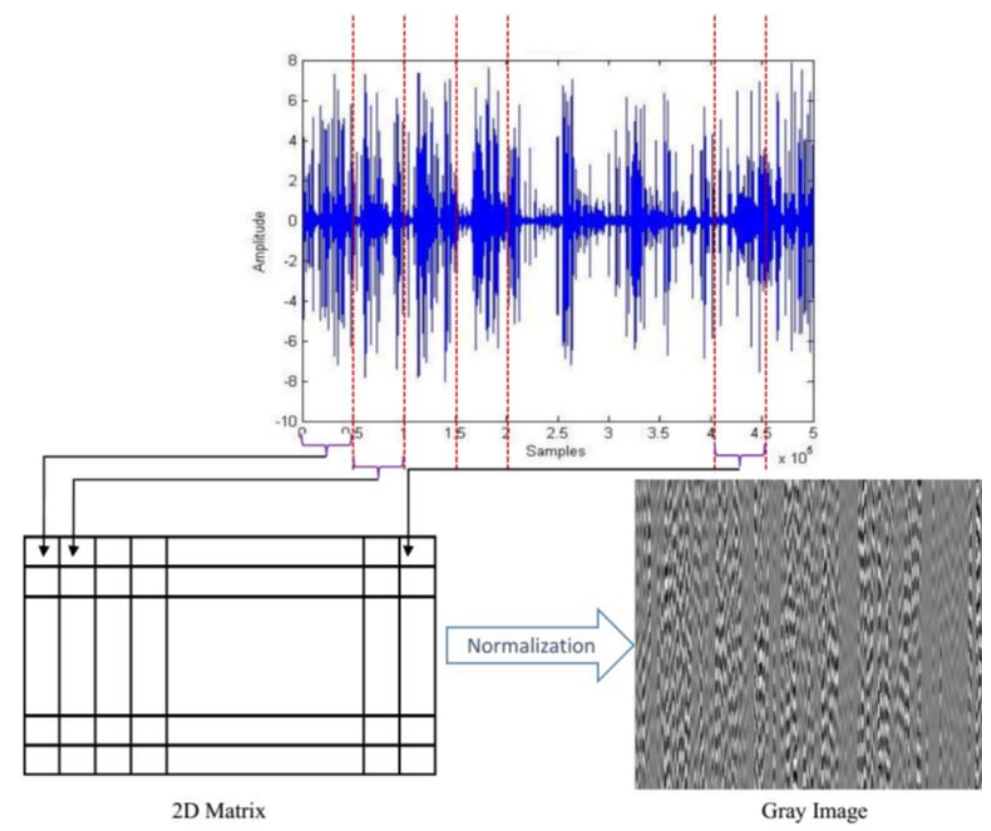

Figure 2. A Detailed Outline of the Conversion Process from a 1D Time-domain Signal to a 2D Gray Image

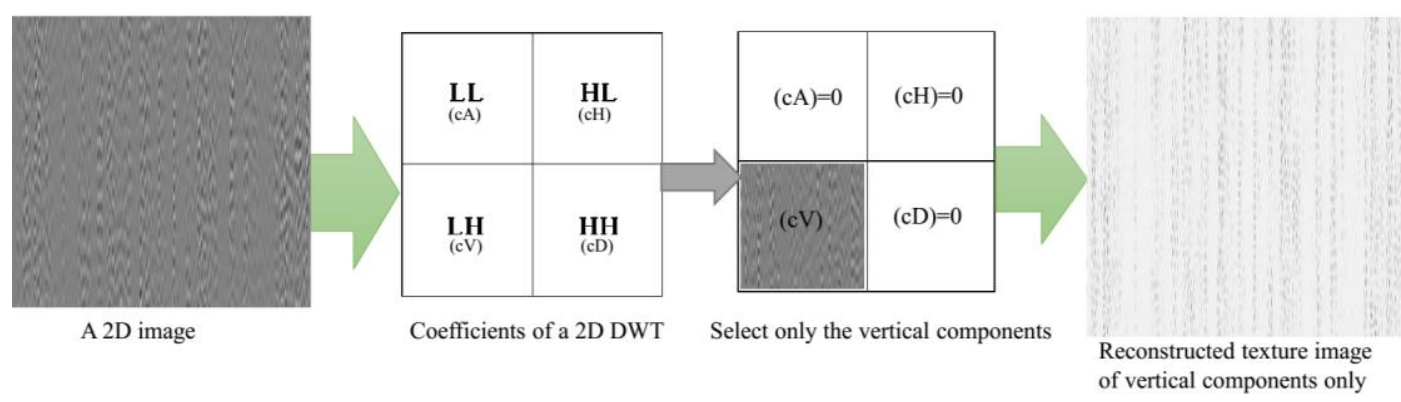

Figure 3. The Reconstruction Procedure of a Texture Image Considering the Vertical Components Only

In order to extract the significant texture of our experimental AE dataset, we apply a 2D-DWT to each converted image, resulting in four distinct combinations of coefficients: approximation (LL), horigontal detail (LH), vertical detail (HL) and diagonal detail (HH) coefficients. As vertical textures are noticeable in numerous experimental AE faults, we select only the vertical detail coefficients in the reconstruction of an image. A detailed outline of an image reconstruction considering only the vertical coefficients is depicted in Figure 3, where all other coefficients are set to 0 .

The possibility of abnormality still exists in reconstructed gray images due to the effects of noise, and these may detract from the reliability of features. In general, for smoothing the gray image, a Gabor filter is directly applied to an image and the features are extracted from the Gabor filtered image [22]. A 2D Gabor filter consists of a complex exponential centered at a given frequency and modulated by a Gaussian envelop. Because 
of the complex exponential, the filtered image has both real and imaginary parts [22-24]. A Gabor filter can be expressed as:

$$
G\left(x, y, k_{x}, k_{y}\right)=\exp \left\{\frac{-(x-X)^{2}+(y-Y)^{2}}{2 \sigma^{2}}\right\} \cdot e^{j\left(k_{x} x+k_{y} y\right)}
$$

where $x$, and ${ }^{y}$ represent the spatial coordinates, $k_{x}$ and ${ }^{k}$ represent the frequency coordinates, and $X, Y$ are the spatial localizations of the Gaussian window. The filter selectivity in spatial frequency and orientation is given by

$$
\omega=2 \pi \sqrt{k_{x}^{2}+k_{y}^{2}} \text { and } \theta=\arctan \frac{k_{x}}{k_{y}} .
$$

In the case of a 2D image, the absolute square of the correlation between an image and the 2D Gabor function provides a local spectral energy density concentrated around a given position and frequency in a certain direction. The Gabor filters are self-similar and all filters can be generated from one mother wavelet via dilation and rotation. Typically, a filter bank consists of Gabor filters, where various scales and rotations are convolved with the signal and resultant a Gabor space. The filtering operation with the Gabor filter can be expressed as:

$$
G(x, y)=I(x, y) * g(x, y)
$$

where $G(x, y)$ denotes the complex convolution result, which can be decomposed into real and imaginary parts. In our experiment, the pixel intensity of the global feature image is used to calculate the feature vectors. The global feature image is calculated using the following equation [25]:

$$
\begin{aligned}
m(x, y) & =\sqrt{k_{c}^{2}(x, y)+k_{s}^{2}(x, y)} \\
\text { where } \quad k_{c}=r e(g(x, y) * i(x, y)), \quad k_{s} & =\operatorname{Im}(g(x, y)) * i(x, y), \quad \text { and } \quad * \text { denotes a }
\end{aligned}
$$
convolution operator.

Image processing is a key research area for various applications in the extraction of detailed information from a natural image. Content-based image retrieval via image segmentation is an effective method to divide an image into a number of different regions [26]; however, conventional segmentation methods are complex and many are problematic in terms of image retrieval. Therefore, to take the benefits of image segmentation and improve feature reliability, in this study we segmented an image into a number of sub-images, as depicted in [27]. We then applied a 2D Gabor filter of equal size on the sub-images and extract the feature vectors from the sub-images. The subimages of an image are calculated using the following formula:

$$
N_{s-i m}=\frac{N_{r-p i x} \times N_{c-p i x}}{\operatorname{size}_{s-i m}^{2}}
$$

where $N_{s-i m}$ is number of sub-images, $N_{r-p i x}$ is the number of row pixels, and $N_{c-p i x}$ is the number of column pixels.

In order to observe the impact of segment size variation on the performance of the proposed model, we consider the following sub-image sizes: $16 \times 16,32 \times 32,64 \times 64$, $128 \times 128$, and $256 \times 256$; where the original image size is $256 \times 256$. It is notable that the number of sub-images varies depending on the segment size. An example scenario of generating 64×64 sub-images from a sample 256×256 image is depicted in Figure 4, 
where the first sub-image is formed by $(1-64) \times(1-64)$ pixels and the last sub-image is formed by (193-256)×(193-256) pixels.

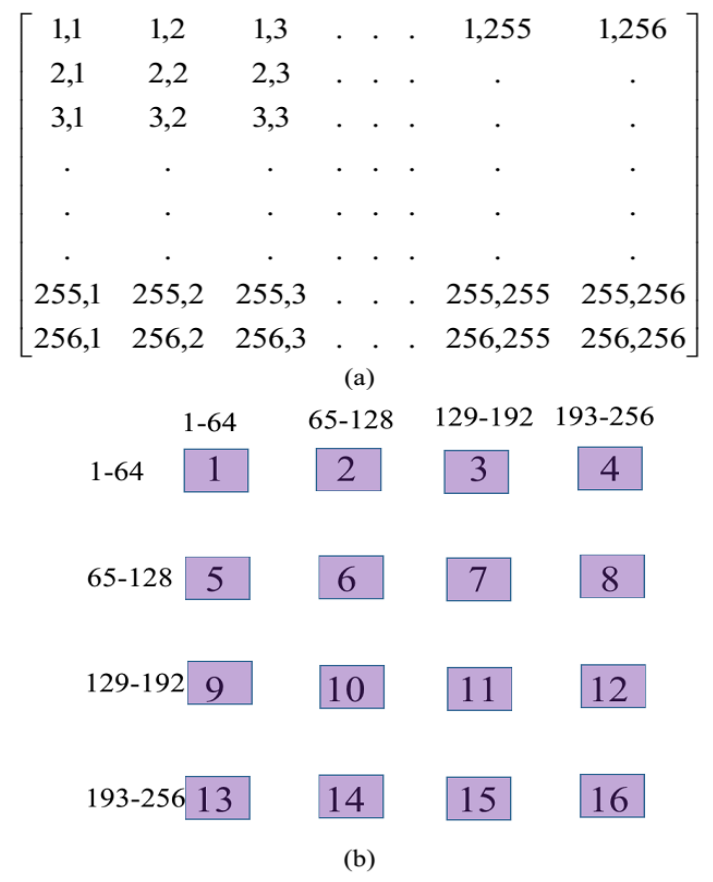

\section{Figure 4. A 256×256 Image: (a) Positions of Pixels in a 2D Space, (b) Sub- Image $(64 \times 64)$ Numbering}

Two important parameters of a Gabor filter, the frequency and orientation angle, have a significant impact on feature vectors. While it is important to set the parameters with optimum values, there is no formal technique for choosing the parameters. Thus, experience-guided intuition, trial and error, or heuristic search is used to select the parameters. In this paper, a bank of Gabor filters with various scales and orientations is applied and the frequency and orientation angle to 2 and $3 \pi / 4$ are selected, respectively, in order to select the distinct texture information of numerous faults. Figure 5 shows sample images of the steps involved in the proposed model, where vertical texture information is more visible after applying Gabor filter.

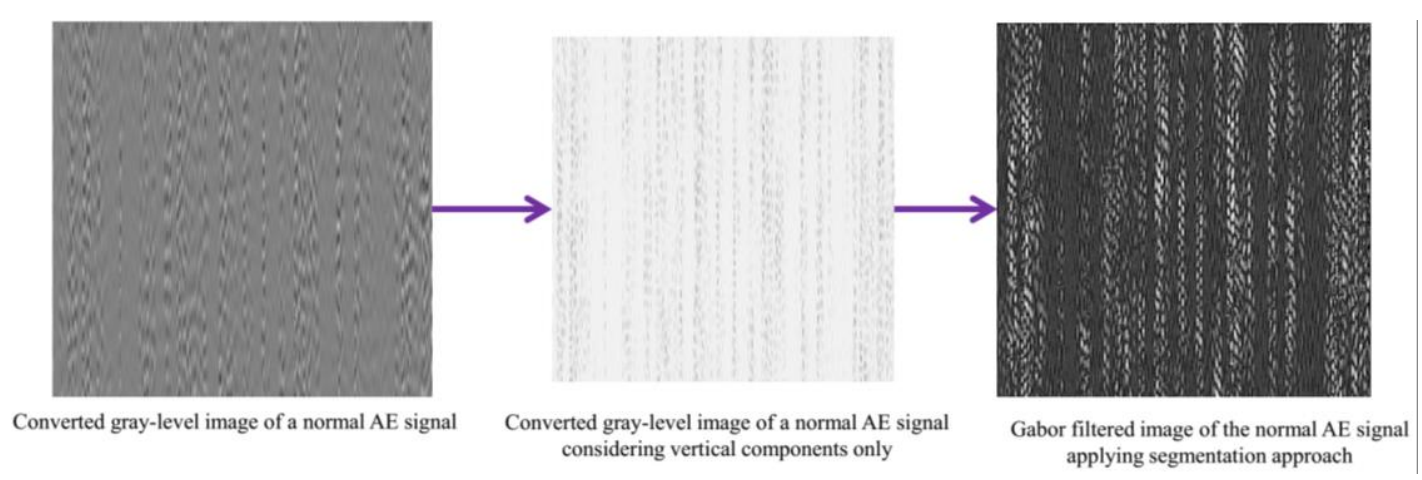

Figure 5. Texture Information of Various Blocks Involved in the Proposed Model 
Numerous statistical parameters are measured in this study, including uniformity and the mean and standard deviation, to construct the final feature vector of the fault signals. Figure 6 shows a sample feature of vectors of numerous faults in a range from 1 to 20, where we experience distinct features for all statistical parameters of Gabor-filtered subimages.

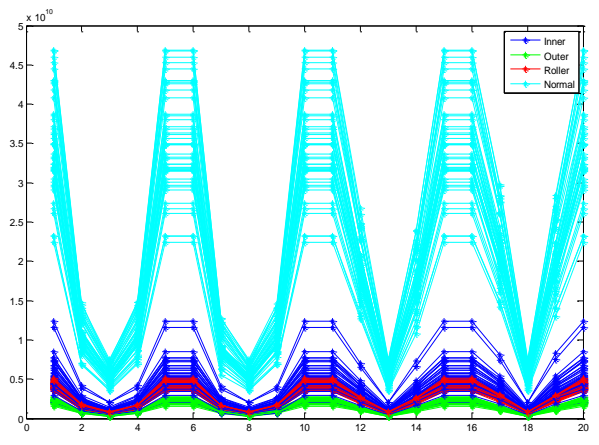

(a)

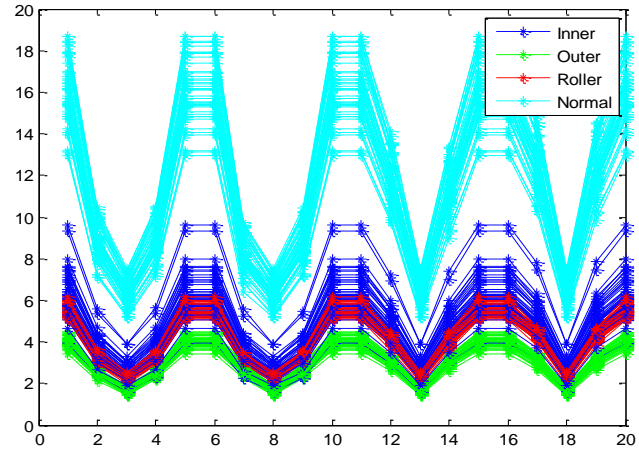

(b)

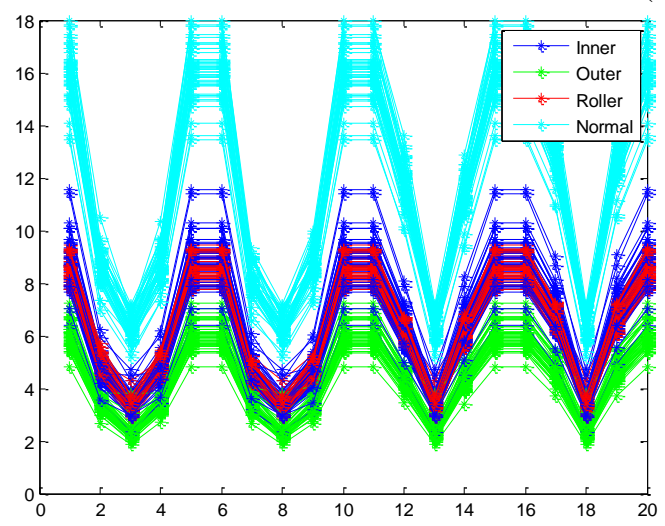

(c)

Figure 6. Sample Features of Various Faults in the Range of 1 to 20: (a) Uniformity, (b) Mean, and (c) Standard Deviation

The SVM separates the test samples into one of the two classes, and consequently, multi-class SVMs (MCSVMs) are utilized to identify multiple bearing defects. In order to design MCSVMs, the following three approaches can be considered: one-against-all (OAA), one-against-one (OAO), and one-acyclic-graph (OAG). The OAA method, one of the most popular and simplest techniques for multi-class classifiers, is employed in this study. In the OAA approach, each SVM structure discriminates one class from the others, and the final decision can be made by selecting the SVM structure that yields the highest output value [7]. As the feature vectors of numerous faults overlap each other, it is not possible to classify using a linear classifier; therefore, in this study we utilize the Gaussian radial kernel function in each SVM due to its simplicity and dynamic non-linear classification capacity. The Gaussian radial basis kernel function is represented as follows:

$$
k\left(s v_{i}-s v_{j}\right)=\exp \frac{\left\|s v_{i}-s v_{j}\right\|^{2}}{2 \sigma^{2}}
$$

where $k\left(s v_{i}, s v_{j}\right)$ is the kernel function, $s v_{i}$ and $s v_{j}$ are the input feature vectors, and $\sigma$ is a parameter set by users to determine the effective width of the basis kernel function. 


\section{Experimental Evaluation}

\subsection{Experimental Acoustic Emission Fault Dataset}

The use of a practical dataset is a considerable issue in the performance evaluation of a proposed model. Therefore, we setup an experiment as depicted in Figure 7, and then collect a dataset by creating physical cracks in various locations of an induction motor bearing. A diamond cutter-bit is used to generate a crack on the surface of the bearing. In general, a number of fault signals such as inner, outer, roller, ball, case, bore, and face may occur at the bearing of an induction motor; however, in our experiment we acquire three single induction motor AE faults, inner, outer, and roller, from the bearing. These signals are directly collected from the AE sensors attached to an induction motor. In addition, a normal bearing is used for benchmarking in this study. As a baseline, a normal signal is collected from the normal bearing of a healthy motor. Table 1 shows a detailed description of seeded bearing defects, and 90 0.5-second AE signals are obtained for each bearing defect sampled at $1 \mathrm{MHz}$ in this study.

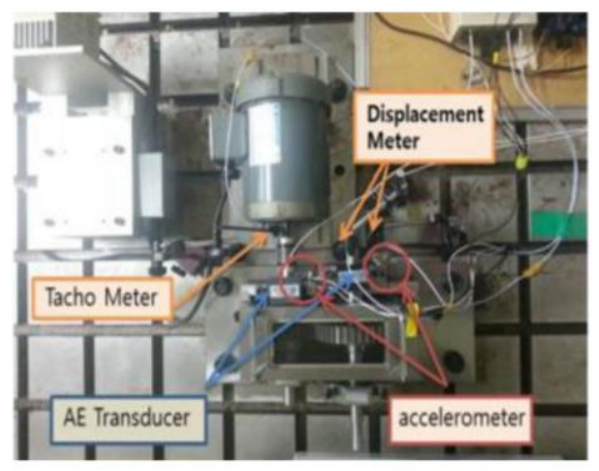

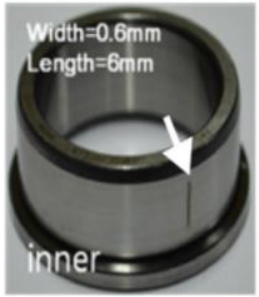
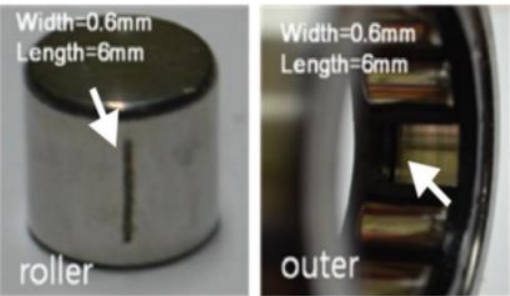

(b)

(a)

Figure 7. (a) A Test Fixture and (b) Numerous Faults Information

Table 1. Statistical Information of Fault Signals Used in the Experiment

\begin{tabular}{l|l}
\hline Parameters & Values \\
\hline Operating frequency & 15 \\
\hline Slim ratio & 1 \\
\hline Rotation per minute & 900 \\
\hline Maximum display frequency & 900 \\
\hline Defect frequency of outer & 78.6285 \\
\hline Defect frequency of inner & 116.3715 \\
\hline Defect frequency of roller & 74.5980 \\
\hline
\end{tabular}

\subsection{Experimental Results and Analysis}

In this study, we evaluate the performance of the proposed model in terms of classification accuracy considering the following statistical parameters: uniformity, mean, and standard deviation. The mathematical expressions of numerous statistical parameters are presented in Table 2. 


\section{Table 2. Mathematical Expressions of Various Statistical Parameters used} in the Experiment

\begin{tabular}{l|l}
\hline Parameter & Mathematical expression \\
\hline Uniformity & $U=\sum_{i} \sum_{j} x_{d}^{2}(i, j)$ \\
\hline Mean & $\mu=\frac{1}{n} \sum_{i} \sum_{j} x(i, j)$ \\
\hline Standard deviation & $\sigma=\sqrt{\frac{1}{n} \sum_{i, j=0}^{N-1}(x(i, j)-\mu)^{2}}$ \\
\hline
\end{tabular}

The classification accuracy is measured with respect to the true positive (TP), where TP is the number of faults in class $i$ that are correctly classified into the class. In order to validate the effectiveness of the proposed segmentation based model, the segment size is varied in the range of 16-256 (/maximum image size). Finally, for numerous segment sizes we obtain different sizes of feature vectors as depicted in Figure 8.

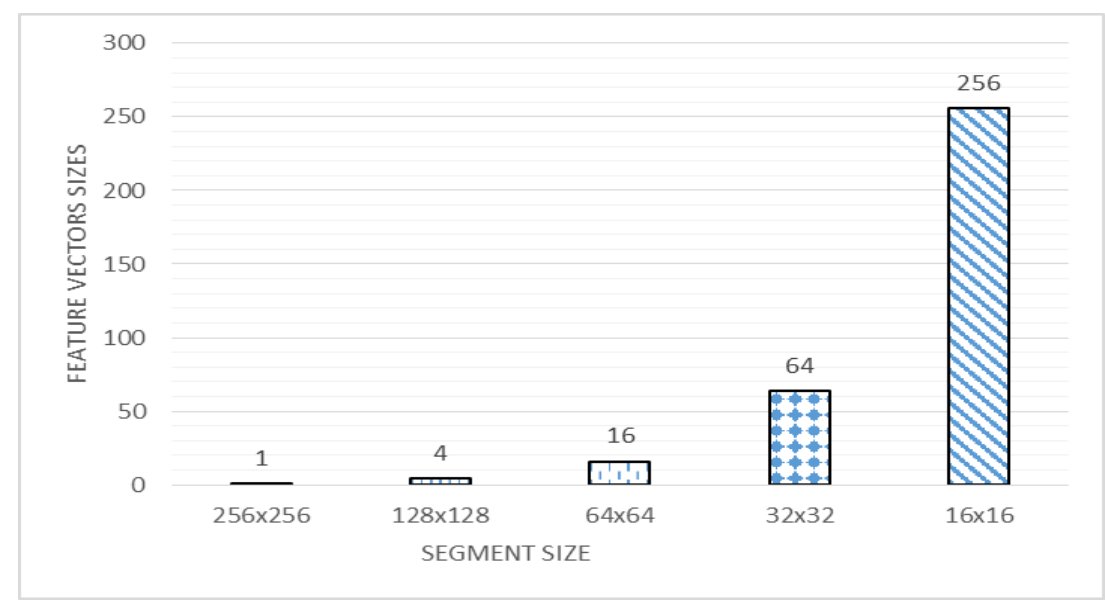

Figure 8. Segment Size versus Feature Vectors Sizes

The optimal selection of the sigma $(\sigma)$ value for the Gaussian radial function is a vital issue in determining the non-linear classification accuracy. In this study, the influence of $\sigma$ value on the performance of the proposed model is evaluated in a range from 0.1 to 2 with an interval of 0.1 for the numerous dimensions to select $\sigma$ values for each SVM. Table 3 illustrates detailed $\sigma$ values of various SVMs for numerous induction motor faults. In addition, we compare the proposed model with the conventional 2D Gaborfilter-based approach in [26] for various sub-image sizes, as shown in Table 4.

Table 3. Optimal Sigma Values of Various SVM Structures

\begin{tabular}{c|c|c}
\hline Faults & Optimal range & Selected values \\
\hline Inner (SVM-1: class 1) & $0.4<\sigma<0.9$ & 0.6 \\
\hline Outer (SVM-2: class 2) & $0.2<\sigma<1.9$ & 1.0 \\
\hline Normal (SVM-3: class 3) & $0.5<\sigma<1.6$ & 1.2 \\
\hline Roller (SVM-4: class 4) & $0.4<\sigma<0.6$ & 0.5
\end{tabular}


Table 4. Average Classification Accuracy of Various Statistical Parameters and Segment Sizes

\begin{tabular}{l|c|c|c|c}
\hline Segment Size & Feature Size & Uniformity & Mean & Standard Deviation \\
\hline $16 \times 16$ & 256 & 91.66667 & 92.22223 & 91.666668 \\
\hline $32 \times 32$ & 64 & 97.22223 & 96.111113 & 93.333335 \\
\hline $\mathbf{6 4 x 6 4}$ & $\mathbf{1 6}$ & $\mathbf{1 0 0}$ & $\mathbf{9 7 . 2 2 2 2 3}$ & $\mathbf{9 3 . 8 8 8 8 8 7}$ \\
\hline $128 \times 128$ & 4 & 94.44445 & 93.88889 & 93.333335 \\
\hline $256 \times 256^{*}$ & 1 & 96.11111 & 93.333333 & 93.333333 \\
\hline
\end{tabular}

* Conventional 2D Gabor filtered method

In Table 4, an image with a segment size of $256 \times 256$ represents the conventional Gabor filtered approach. The experimental results demonstrate that the conventional approach shows comparatively lower performance than the proposed segmentation-based approach in various segment sizes. This improved result is due to the extraction of detailed and reliable feature vectors from the proposed model compare. However, the selection of optimal segmentation size is a vital issue in the proposed model and can control the classification accuracy. We consider various segment sizes to evaluate the classification accuracy of the proposed model. It is expected that classification accuracy will increase with the increment of feature size; however, for our dataset we experience less classification accuracy for small and large features. We also observe that maximum classification accuracy is obtained for $64 \times 64$ segments when the feature vector size is 16 . The size of the feature vector is small for large segments, which cannot contain meaningful information of a fault. In contrast, the feature vector is significantly larger for small segments, increasing the computational complexity of the classifier as well as the overall model, which decreases the classification accuracy.

\section{Conclusions}

In this paper, we proposed a new diagnosis methodology composed of signal conversion, feature calculation, feature dimension selection, and classification to achieve highly reliable fault diagnosis performance. We first converted a 1D time-domain signal to a $2 \mathrm{D}$ texture image, segmented the resulting image into a number of sub-images based on segment size, and calculated the feature vectors. Finally, we identified multiple bearing defects by employing the individually-trained OAA MCSVMs to maximize the classification ability of the standard OAA-MCSVMs. The experimental results indicate that the proposed method outperforms the conventional Gabor-filter-based approach, yielding an average classification accuracy of $97 \%$ with segment sizes less than $64 \times 64$.

\section{Acknowledgments}

This work was supported by the National Research Foundation of Korea (NRF) grant, funded by the Korean government (MSIP) (No. NRF-2015K2A1A2070866).

\section{References}

[1] A. G. Rhorn, E. Sejdic and J. Jiang, "Fault diagnosis in machine tools using selective regional correlation”, Journal of Mechanical Systems and Signal Processing, vol. 20, (2006), pp. 1221-1238.

[2] A. Sadeghian, Z. Ye and B. Wu, "Online Detection of Broken Rotor Bars in Induction Motors by Wavelet Packet Decomposition and Artificial Neural Networks", IEEE Transactions on Instrumentation and Measurement, vol. 58, no. 7, (2009), pp. 2253-2263.

[3] V. T. Tran, F. AlThobiani, A. Ball and B. K. Choi, "An application to transient current signal based induction motor fault diagnosis of Fourier-Bessel expansion and simplified fuzzy ARTMAP", International Journal of Expert Systems with Application, vol. 40, (2013), pp. 5372-5384. 
[4] H. Keskes, A. Braham and Z. Lachiri, "Broken rotor bar diagnosis in induction machines through stationary wavelet packet transform and multiclass wavelet SVM", International Journal of Electric Power Systems Research, vol. 97, (2013), pp. 151-157.

[5] A. Bouzida, O. Touhami, R. Ibtiouen, A. Belouchrani, M. Fadel and A. Rezzoug, "Fault Diagnosis in Industrial Induction Machines Through Discrete Wavelet Transform", IEEE Transactions on Industrial Electronics, vol. 58, no. 9, (2011), pp. 4385-4395.

[6] M. Kang and J. Kim, "Singular Value Decomposition Based Feature Extraction Approaches for Classifying Faults of Induction Motors", International Journal of Mechanical Systems and Signal Processing, vol. 41, no. 1-2, (2013), pp. 348-356.

[7] D. Nguyen, M. Kang, C.H. Kim and J. Kim, "Highly Reliable State Monitoring System for Induction Motors Using Dominant Features in a 2-Dimension Vibration Signal”, New Review of Hypermedia and Multimedia, vol. 13, no. 3-4, (2013), pp. 245-258.

[8] Z. Yanbing, L. Yibing, X. Weidong and H. Jiantao, "Gear fault feature extraction based on the combination of Gabor reconstruction and bispectral analysis", Proceeding of the 31st Chinese Control Conference (CCC), (2012); Hefei, China.

[9] H. Li, H. Zheng and L. Tang, "Gear Fault Detection Based on Teager-Huang Transform", International Journal of Rotating Machinery, vol. 502064, (2010), pp. 1-9.

[10] G. F. Bin, J. J. Gao, X. J. Li and B. S. Dhillon, "Early fault diagnosis of rotating machinery based on wavelet packets-Empirical mode decomposition feature extraction and neural network", Interntional Journal of Mechanical Systems and Signal Processing, vol. 27, (2012), pp. 696-711.

[11] Z. Sadeg, Y. Wang and W. Kesheng, "Fault diagnosis and prognosis using wavelet packet decomposition Fourier transform and artificial neural network", Journal of Intelligent Manufacturing, vol. 24, no. 6, (2013), pp. 1213-1227.

[12] L. Gao, F. Zai, S. Su, H. Wang, P. Chen and L. Liu, "Study and Application of Acoustic Emission Testing in Fault Diagnosis of Low-Speed Heavy-Duty Gears", Journal of Sensors, vol. 11, (2011), pp. 599-611.

[13] T. D. Van and C. U-Pil, "Signal Model-Based Fault Detection and Diagnosis for Induction Motors Using Features of Vibration signal in Two-Dimension Domain", Journal of Mechanical Engineering, vol. 57, no. 9, (2011), pp. 655-666.

[14] J. Uddin, D. Nguyen and J. M. Kim, "A Reliable Fault Detection and Classification Model of Induction Motors using Texture Features and Multi-class Support Vector Machines", Journal of Mathematical Problems in Engineering, vol. 2014, (2014), pp. 1-9.

[15] W. C. Jang, Y. H. Park, M. S. Kang and J. M. Kim, "Mechanical Fault Classification of an Induction Motor using Texture Analysis", Journal of the Korea Scoiety of Computer and Information, vol. 18, no. 12, (2013), pp. 12-19.

[16] M. R. Shahriar, T. Ahsan and U. P. Chong, "Fault diagnosis of induction motors utilizing local binary pattern-based texture analysis", EURASIP Journal on Image and Video Processing, vol. 2013, no. 29, (2013), pp. 1-11.

[17] J. Uddin, R. Islam and J. M. Kim, "Texture Feature Extraction Techniques for Fault Diagnosis of Induction Motors", Journal of Convergence, vol. 5, no. 2, (2014), pp. 15-20.

[18] M. Kang and J. M. Kim, "Reliable Fault Diagnosis of Multiple Induction Motor Defects Using a TwoDimensional Representation of Shannon Wavelets", IEEE Transactions on Magnetics, (2014), pp. 1-13.

[19] M. Idrissa and M. Acheroy, "Texture classification using Gabor filters", Pattern Recognition letters, vol. 23, (2002), pp. 1095-1102.

[20] L. Weitao, M. Kezhi, Z. Hong and C. Tianyou, "Designing compact Gabor filter banks for efficient texture feature extraction", Proceeding of the 11th International Conference on Control Automation Robotics \& Vision (ICARCV), (2010).

[21] T. Yu, M. Vallipuram, V. Brijesh and B. Michael, "A Texture Feature Extraction Technique Using 2DDFT and Hamming Distance", Proceeding of the Fifth International Computational Intelligence and Multimedia Applications (ICCIMA), (2003); Xi'an, China.

[22] S. Lahmiri and M. Boukadoum, "Hybrid Discrete Wavelet Transform and Gabor Filter Banks Processing for Features Extraction from Biomedical Images", Journal of medical Engineering, (2013) pp. $1-13$

[23] F. Liu, "Texture identification using Gabor wavelet based signal processing", Institute of Technology and Innovation, university of Southern Denmark, (2011), pp. 1-5.

[24] D. Barina, "Gabor Wavelets in Image Processing", Proceedings of conference and competitions student (EEICT), (2011); Guangzhou, China.

[25] J. Wang and A. K. Asundi, "A computer vision system for wineglass defect inspection via Gabor-filterbased texture features", International Journal of Information Science, (2000), pp. 157-171.

[26] I. Mahamadou and A. Marc, "Texture classification using Gabor filters", Pattern Recognition letters, vol. 23, (2002), pp. 1095-1102.

[27] A. Noora and K. Mohammed, "A proposed approach for finding the percentage of similarity between images", International Journal of Video and Image processing and Network Secuirity, vol. 12, no. 4, (2012), pp. 14-17. 


\section{Authors}

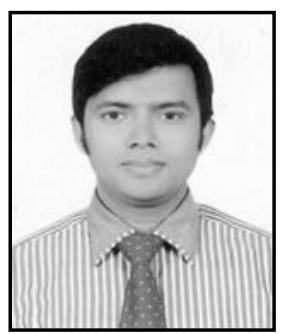

Jia Uddin, He received a BSc. degree in Computer and Communication Engineering from the International Islamic University, Chittagong (IIUC), Bangladesh, in 2005, an MS degree in Electrical Engineering with an emphasis on Telecommunications from the Blekinge Institute of Technology (BTH), Sweden, in 2010. He completed his Ph.D. (Computer Engineering) at the University of Ulsan (UoU), South Korea in 2015. He is an Assistant Professor in the Department of Computer Science and Engineering at BRAC University, Bangladesh. His research interests include Fault Diagnosis, Parallel Computing, and Wireless Networks. He is a member of the IEB and the IACSIT.

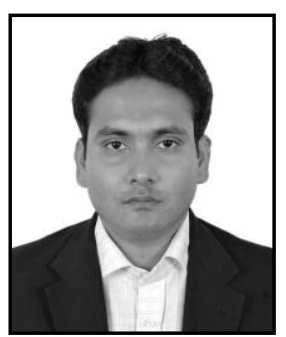

Md. Rashedul Islam, He is a Ph.D. student in the Department of Electrical, Electronic, and Computer Engineering at the University of Ulsan (UoU), South Korea. He is an Assistant Professor at the Department of Computer Science and Engineering in the University of Asia Pacific (UAP), Bangladesh. He has completed a BS (Hons.) in Computer Science and Engineering at the University of Rajshahi, Bangladesh in 2006 and an M.Sc. in Informatics from Högskolan i Borås (University of Boras), Sweden in 2011. He is a researcher as well as an information system analyst. His current research interest is motor faults signal analysis, parallel algorithm/processing, and GPS.

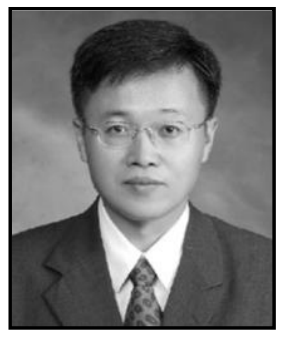

Jong-Myon Kim, He received a BS in electrical engineering from Myongji University, Yongin, Korea, in 1995, an MS in electrical and computer engineering from the University of Florida, Gainesville, in 2000 , and a $\mathrm{PhD}$ in electrical and computer engineering from the Georgia Institute of Technology, Atlanta, in 2005. He is an associate professor of Electrical Engineering at the University of Ulsan, Korea. His research interests include multimedia processing, multimedia specific processor architecture, parallel processing, and embedded systems. He is a member of IEEE and IEEE Computer Society.

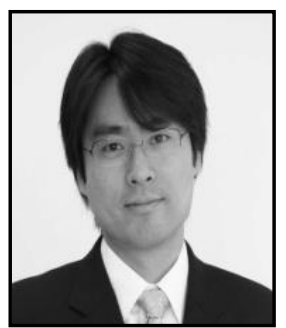

Cheol-Hong Kim, He received B.S., M.S., and Ph.D. degrees in Computer Engineering from Seoul National University, Seoul, Korea, in 1998, 2000, and 2006, respectively. He is currently an associate professor of Electronics and Computer Engineering at Chonnam National University, Gwangju, Korea. His current research interests include embedded systems, mobile systems, system on chip design, and parallel processing. 